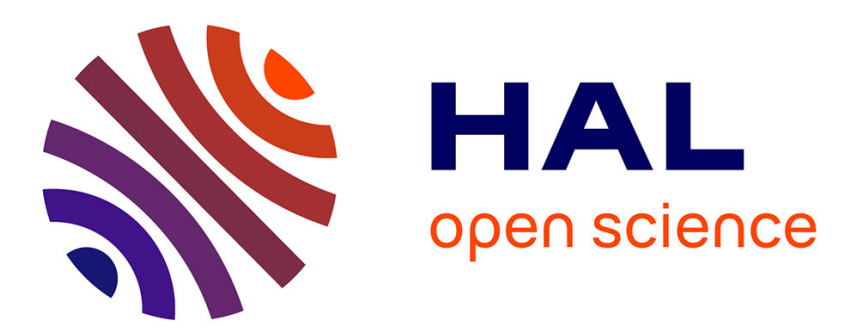

\title{
Entre occultation volontaire et régulation publique: les petites morts de la critique radicale des sciences
}

Céline Pessis, Sara Angeli Aguiton

\section{To cite this version:}

Céline Pessis, Sara Angeli Aguiton. Entre occultation volontaire et régulation publique: les petites morts de la critique radicale des sciences. Écologie \& politique: sciences, culture, société, 2015. hal-02009609

\section{HAL Id: hal-02009609 \\ https://hal.science/hal-02009609}

Submitted on 18 Feb 2019

HAL is a multi-disciplinary open access archive for the deposit and dissemination of scientific research documents, whether they are published or not. The documents may come from teaching and research institutions in France or abroad, or from public or private research centers.
L'archive ouverte pluridisciplinaire HAL, est destinée au dépôt et à la diffusion de documents scientifiques de niveau recherche, publiés ou non, émanant des établissements d'enseignement et de recherche français ou étrangers, des laboratoires publics ou privés. 

et régulation publique : les petites morts de la critique radicale des sciences. », Ecologie \& politique. 2015/2, n 51, p. 93-105.

\title{
Entre occultation volontaire et régulation publique : les petites morts de la critique radicale des sciences
}

\author{
Céline Pessis et Sara Angeli Aguiton
}

\begin{abstract}
Résumé - Si la critique (et l'autocritique) des sciences a une histoire articulée à celle de l'écologie politique, son héritage est aujourd'hui invisible. Comment expliquer l'absence de transmission, entre générations de chercheurs comme au sein de la mouvance écologiste, de la mémoire de ce mouvement de critique radicale des sciences des années 1970 ? Après avoir éclairé les formes de l'engagement critique des scientifiques durant l'entre-deux-mai (1968-1981), cet article propose quelques pistes afin de rendre compte des reconfigurations sociales, politiques et institutionnelles qui ont produit une telle occultation. L'étude de deux moments est privilégiée : la reprise en main politicoindustrielle de la recherche au tournant des années 1980, qui vient offrir aux chercheurs un nouvel ethos scientifique mêlant vulgarisation et innovation ; et le tournant réformiste " sciences-société " des années 2000, qui, tout en poursuivant une régulation libérale des innovations technocapitalistes, entend gérer le renouveau contestataire par l'inclusion de la société civile.
\end{abstract}

mots Clés - Technosciences, contestations, politiques de recherche, engagement, institutionnalisation. abstRaCt - Since its early days, the political ecology is entangle with a (self)critique of science. However, the legacy of the radical critic of science from the 1970s is today invisible in the cultural transmission among researchers and within the ecologist movement. How can we explain it? This paper aims to bring some answers by describing the critical political engagement of scientists between 1968 and 1981 and exploring the social, political and institutional reorganizations which have produced such occultation. The study of two moments is conducted: the political and industrial takeover of research in the 1980s, which gave birth to a new scientific ethos made of mediation and innovation; and the "science and society" reformist movement of the 2000s which, pursuing a liberal regulation of technocapitalist innovations, aims to manage the protest resurgence by including civil society in its apparatus.

KeywoRDs - Technosciences, contestations, research politics, engagement, institutionalization.

Beaucoup ont découvert Alexandre Grothendieck à l'occasion de l'hommage qui lui fut rendu au moment de son décès le 13 novembre 2014. La communauté mathématique s'enorgueillit alors de ce "génie national », tout en se gardant bien souvent d'évoquer les raisons de son retrait de la recherche au début des années 1970. Alors que l'héritage scientifique de Grothendieck est en passe de se rouvrir, on ne peut que s'interroger sur le silence ou l'incompréhension qui entourent son engagement dans le mouvement de critique radicale des sciences.

Si la personnalité hors normes de Grothendieck se prêta rarement à l'action collective, à l'exception notable du groupe Survivre et Vivre qu'il fonda en 1970, il participa pourtant pleinement de toute une culture critique et d'un des mouvements contestataires parmi les plus subversifs - que les hommages rendus aujourd'hui au mathématicien semblent enterrer à nouveau. Plutôt que 
d'opposer l'individualisme de Grothendieck à l'écologisme associatif de Serge Moscovici, décédé deux jours après lui ${ }^{1}$, nous voudrions ainsi rappeler la proximité militante qui lia les deux hommes et qui amena Moscovici à situer ce mouvement de critique radicale de la science comme première filiation intellectuelle du mouvement écologiste.

Par certains aspects, le paysage de la recherche n'a guère changé et l'on comprend qu'il serait de mauvais ton de se remémorer qu'Alexandre Grothendieck démissionna avec fracas de son institut de recherche pour cause de financements militaires. Au-delà pourtant de ces continuités, l'invisibilité de l'héritage critique de Grothendieck (en dehors de petits milieux décroissants) questionne. Comment expliquer l'absence de transmission, entre générations de chercheurs comme au sein de la mouvance écologiste, de la mémoire de ce mouvement de critique radicale des sciences des années 1970 ? Après avoir éclairé les formes de l'engagement critique des scientifiques durant l'entre-deux-mai (1968-1981), cet article propose quelques pistes afin de rendre compte des reconfigurations sociales, politiques et institutionnelles qui ont conduit aujourd'hui à une telle occultation. L'étude de deux moments, qui s'articulent à deux types de gestion de la critique de la science, est privilégiée : la reprise en main politico-industrielle de la recherche au tournant des années 1980, qui vient offrir aux chercheurs un nouvel ethos scientifique mêlant vulgarisation et innovation; et le tournant réformiste « sciences-société " des années 2000 qui, tout en poursuivant une régulation libérale des innovations technocapitalistes, entend gérer le renouveau contestataire par l'inclusion de la société civile.

\section{La big science contestée : critique radicale et engagement social}

Le mouvement de critique radicale de la science émerge de l'opposition gauchiste à la guerre du Viêt Nam et du processus de " délégitimation des intellectuels » ouvert par Mai $68^{2}$. Il débute par la dénonciation des liens organiques unissant scientifiques, militaires et industriels. Celles et ceux qui, en mal d'idéalisme et de marginalisation élitaire, sont entrés en science " comme on entre au monastère » en viennent à dénoncer la massification, l'industrialisation et la militarisation de la recherche ${ }^{3}$. C'est la fin de l'innocence des scientifiques et de l'âge d'or de la science, ce temps où « le "savant" cherchait la "vérité" sur le plan théorique et, sur le plan social, contribuait à améliorer le sort de l'humanité ${ }^{4} \gg$.

En analysant le rôle de la science dans la domination impérialiste ou dans l'exploitation ouvrière, ces scientifiques critiques invitent à considérer cette

\footnotetext{
1. P. Piro, «Yves Cochet : "Serge Moscovici et Alexander Grothendieck ont créé le déclic en moi" », Politis, n 1328, 20-26 novembre 2014.

2 . Pour une analyse et des références plus détaillées sur ce mouvement, $c f$. « Petit panorama de la critique des sciences des années $1970 »$, dans C. Pessis (dir.), Survivre et Vivre. Critique de la science, naissance de l'écologie, L'Échappée, Montreuil, 2014, p. 343-360.

3 . J. Feldman, «La science en mutation», dans M. Armatte (dir.), Le sujet et l'objet. Confrontations. Séminaire d'interrogations sur les savoirs et les sciences, Éditions du CNRS, Paris, 1984.

${ }^{4}$. P. Thuillier, «Y a-t-il une science innocente ? », La Recherche, 8 janvier 1971, p. 7-8.
} 
dernière comme un instrument privilégié du capitalisme moderne et à la replacer au centre des luttes. Ancrés dans diverses institutions scientifiques et surtout dans l'Université, ils se veulent ainsi parties prenantes de mouvements sociopolitiques plus larges : des luttes « traditionnelles 》 (luttes ouvrières, combats anti-impérialistes) aux combats émergents (luttes pour la santé et les conditions de travail, mouvement de libération des femmes, écologie). Sous l'influence du mouvement environnementaliste nord-américain, et en France à travers l'engagement écologiste de Survivre et Vivre, la critique des sciences intègre également celle des technologies, participant alors de l'émergence du mouvement antinucléaire.

On voit ainsi des experts en planification rejeter « la "rationalité" de technocrates » pour prôner « la fusion avec la lutte de ceux qui, commerçants ou paysans, se révoltent contre leur liquidation justifiée précisément par cette "rationalité" ${ }^{5}$ "! Mais, aussi virulente soit-elle, la critique portée par ces « travailleurs scientifiques » ne verse jamais dans la diabolisation. Si, selon leurs analyses, la science tient un tel rôle dans les guerres impérialistes menées à l'encontre du tiers monde, dans la course à la puissance entre les deux blocs, la destruction et l'artificialisation de la nature, ou encore la production de sociétés bureaucratiques et inégalitaires, c'est qu'elle relève moins de l'ordre de la raison ou de l'objectivité, que du social, du politique et de l'économique. C'est à une telle démystification de l'activité scientifique que se livrent en premier lieu ces contestataires. Loin de toute métaphysique, elle s'ancre dans l'analyse des conditions de travail dans les milieux scientifiques.

Certains mettent en lumière l'exploitation qui sévit dans les laboratoires comme dans les usines, la parcellisation et la hiérarchisation du travail liées aux gros appareillages techniques qui font de la recherche une quasi-industrie. Et tous de se demander : comment distinguer encore la « science » de ses " applications techniques » lorsqu'elle atteint une telle visée instrumentale ? D'autres mettent en lumière les désaccords et les doutes desdits " experts ", leur étroite spécialisation qui les rend inaptes à saisir la diversité mouvante du vivant ou à maîtriser les mégasystèmes techniques dans leur intégralité. Un droit d'inventaire s'exerce sur la plupart des disciplines : « La géographie, ça sert d'abord à faire la guerre ${ }^{6} » ;$ l'anthropologie, à la colonisation et à faire la guerre ; les mathématiques, à la sélection des étudiants, ainsi qu'au calcul des trajectoires des bombes; quant aux derniers venus, l'informatique et le nucléaire, ne sont-ils pas les enfants chéris de la dernière guerre ? Alors, vraiment, s'interrogent ces scientifiques, n'aurions-nous affaire qu'à de " mauvaises applications » d'une science " bonne et pure » par nature ? Ne serait-ce pas plutôt le système même de production des savoirs qui serait partie intégrante du complexe militaro-industriel ? La guerre du Viêt Nam, qui offre de vastes débouchés à l'industrie chimique et sert de terrain d'expérimentation à la nouvelle microélectronique, ne vient-elle pas en fournir une énième démonstration? Y regardant de près, certains découvrent que les liens entre recherche et armée n'ont rien d'anodins, mais sont au contraire profondément institutionnalisés, notamment par des contrats entre laboratoires et militaires.

\footnotetext{
5 . «La longue marche des cadres intellectuels », Labo-Contestation, n 2, 1971, p. 47.

${ }^{6}$. Selon le titre d'un livre d'Yves Lacoste, La géographie, ça sert d'abord à faire la guerre, La Découverte, Paris, 2014 [1976].
} 
Cette (auto)critique, les scientifiques français la mènent dès l'automne 1968 au sein du principal syndicat des chercheurs (le Syndicat national des chercheurs scientifiques, SNCS), puis par de nombreuses grèves et à l'aide de plusieurs revues qui marquent leur jonction avec d'autres mouvements contestataires. Labo-Contestation, l'une de ces revues, remet ainsi en cause la fonction des scientifiques dans tous les secteurs de la société (laboratoires et bureaux d'études, entreprises, secteur médical et agriculture). Par là, elle travaille à politiser et à soutenir la lutte, non seulement des chercheurs, mais aussi des techniciens et des ingénieurs. Des vétérinaires et des techniciens agricoles analysent par exemple leur rôle dans la mise en place d'une agriculture capitaliste et la sélection des agriculteurs (par l'octroi de subventions ou la « promotion » de technologies adaptées exclusivement aux grandes exploitations). Certains refusent alors un travail au service des grandes firmes intégratrices, préférant rechercher des voies neuves de collaboration avec des groupements d'agriculteurs. Des travailleurs du secteur de la santé s'emploient, eux, à démythifier l'autorité médicale afin de rendre aux malades le pouvoir sur leur corps et leur santé. Les membres du Groupe Information Santé et du Collectif Amiante de Jussieu apportent une aide directe aux ouvriers de l'amiante, du plomb, de l'aluminium, etc., en lutte pour l'amélioration de leurs conditions de travail. Étudiants et médecins participent alors à des enquêtes ouvrières afin de restituer à la maladie son caractère collectif et son sens politique.

Dans une veine proche, bien que plus théorique, la revue Survivre... et Vivre s'en prend au scientisme, cette croyance en la toute-puissance de la science, dénonçant son fantasme de maîtrise du vivant et ses vaines promesses de résolution de la crise écologique dont il est pourtant à l'origine. À la revue s'adjoignent des groupes locaux, et ce petit mouvement de scientifiques critiques se transforme vite en "laboratoire idéologique de la révolution écologique française ${ }^{7} \gg$. Sa critique de la science et des experts devient une ressource clé pour toute une gamme de mouvements contestant l'autorité scientifique et la raison technique (agrobiologistes, antimilitaristes et objecteurs de conscience, comités antinucléaires, etc.).

Institutionnellement, cette révolte des scientifiques prend corps dans les sciences dites « dures » autour de la dénonciation du complexe scientificomilitaro-industriel. Marquant l'entrée dans l'ère des technosciences, elle conteste la distinction entre une science pure et noble, se déroulant dans le ciel éthéré des idées, et ses applications bassement matérielles et souvent néfastes. Elle gagne plus timidement les spécialistes des sciences humaines et sociales, qui, à l'apogée du structuralisme, se montrent plus réticents à contester la scientificité à laquelle ils accédaient enfin. De la focale militaire, la critique s'élargit rapidement pour traquer les multiples façons dont sciences et pouvoirs dominants (bourgeois, impérialistes, technocratiques et modernisateurs, biocapitalistes, patriarcaux) se renforcent et se perpétuent mutuellement. Le dogmatisme de l'enseignement scientifique, puis de la vulgarisation, retient particulièrement l'attention. Au milieu des années 1970, les séminaires critiques se transforment en enseignements « science et société » alliant enquêtes dans les laboratoires et discussions sur les financements et

${ }^{7}$. P. Fournier, Charlie Hebdo, n 86, 10 juillet 1972, p. 10. 
les finalités de la recherche, sur les dogmes scientifiques dominants, ou encore sur le culte de la science et des experts.

La question de l'arrêt ou de la continuation de la recherche traverse de façon lancinante ce mouvement de critique radicale des sciences. Si pour certains, jeunes ingénieurs et physiciens en particulier, la désertion vient y répondre, la plupart d'entre eux se retrouvent autour de l'affirmation d'une responsabilité sociale et environnementale de leur travail. Ce mouvement se caractérise en effet d'abord par son engagement auprès des mouvements sociaux ${ }^{8}$ et sa volonté de transformer le mode de production des savoirs, les structures et les axes de recherche.

À la fin de la décennie, le mouvement des boutiques de sciences inspiré des Pays-Bas se présente comme un outil permettant de prolonger et de pérenniser ces différentes initiatives. Pour le Comité de liaison et d'information sur la santé et les conditions de travail (CLISACT) ${ }^{9}$, une boutique de sciences se conçoit comme un « observatoire populaire de science ", inséré localement dans un réseau militant et incitant à l'ouverture de nouvelles pistes de recherche à partir de la demande populaire ${ }^{10}$. Une telle perspective est défendue lors des assises de l'enseignement supérieur qui se tiennent à l'arrivée de la gauche au gouvernement. Générant de fortes attentes, ce changement politique semble en effet " particulièrement propice pour passer de la critique à l'action constructive ${ }^{11} »$.

\section{« Technoparade ${ }^{12}$ » et enlisement de la critique}

Si les premières années de la décennie 1980 sont marquées par une certaine reconnaissance institutionnelle des mouvements précédents, celle-ci ne s'accompagne pourtant d'aucune transformation des institutions et des orientations de recherche. La " demande sociale » à laquelle les chercheurs sont invités à s'ouvrir s'incarne principalement dans les exigences industrielles et l'impératif de combler le manque de culture scientifique de chacun. Visant explicitement à contrer l'« irrationalisme » et les " mouvements antiscience » de la décennie précédente ${ }^{13}$, les réformes des années 1980 contribuent à la formation d'une nouvelle génération de scientifiques, tandis que sur les décombres du marxisme le biologisme, la complexité et le réseau s'érigent en nouvel esprit scientifique, semblant éloigner la big science et ses contestations.

\footnotetext{
8 . Pour ses jonctions avec les mouvements syndicaux, $c f$. R. Bécot et C. Pessis, «Improbables mais fécondes : les rencontres entre scientifiques critiques et syndicalistes dans les années 1968 », Mouvements, vol. 4, n 80, 2014, p. 51-66.

9 . Le CLISACT (1975-1983) participe aux côtés de syndicalistes à des luttes contre les maladies professionnelles et les pollutions industrielles.

${ }^{10}$. «Propositions en matière de recherche scientifique et populaire », Lutte Santé Sécurité, n 20 , juin 1982, p. 24-25.

${ }^{11}$. J. Stewart et V. Havelange, «Les boutiques de sciences en France : un bilan », Alliage, $\mathrm{n}^{\circ}$ 1, mai 1989, p. 3.

12. F. Cusset, La Décennie. Le grand cauchemar des années 1980, La Découverte, Paris, 2008.

${ }^{13}$. P. Petitjean, «La critique des sciences en France », Alliage, n 35-36, 1998, $<$ www.tribunes.com/ tribune/alliage/35-36/06petit.htm>.
} 
Le nouveau gouvernement s'emploie à enrôler ingénieurs et scientifiques critiques au sein de plusieurs agences spécialisées, mais celles-ci sont rapidement dépourvues de leurs moyens ou détournées de leur fonction. L'investissement dans les arènes institutionnelles participe alors de la canalisation des énergies militantes, lesquelles se trouvent rapidement cantonnées dans un rôle de contre-expertises peu influentes. L'exemple le plus symptomatique est celui de l'énergie ${ }^{14}$. À l'Agence française de maîtrise de l'énergie (AFME), l'objectif de substituer au nucléaire des énergies renouvelables est contrarié dès 1982 par le reniement des engagements présidentiels. Contrastant avec ses formes basistes de la décennie précédente, l'opposition au nucléaire se technicise et se confine au sein d'instances d'information locales, avant de créer, dans l'après-Tchernobyl, des organismes indépendants de recherche tels que la Commission de recherche et d'information indépendantes sur la radioactivité (CRIIRAD).

Au contraire de la plupart des réseaux militants qui périclitent au seuil des années 1980, la nouvelle gauche paysanne et l'agriculture biologique poursuivent, elles, leur essor et leur compagnonnage avec de nombreux scientifiques critiques. Impliqués dans des recherches-actions et le lancement de nouvelles revues, ces derniers participent de l'émergence et de la circulation de nouvelles pratiques du métier ${ }^{15}$. À côté de facteurs internes au domaine agricole, peutêtre faut-il voir dans le dynamisme de ces rencontres le contrecoup positif de leur isolement institutionnel. Malgré sa reconnaissance par l'État en 1980, l'agriculture biologique ne bénéficie en effet de soutien ni financier ni scientifique. Les savoirs qui la soutiennent se structurent et se transmettent au sein de réseaux de praticiens-chercheurs indépendants, tels que l'école de Beaujeu ${ }^{16}$. La recherche d'une pratique paysanne plus autonome se déroule elle aussi en marge des institutions, au sein de groupes de travail associatifs comme le Centre d'étude pour un développement agricole plus autonome (CEDAPA) en Bretagne. Si la pénurie énergétique et la contestation écologique avaient semblé engager l'Institut national de recherche agronomique (INRA) dans une remise en cause de son modèle de développement productiviste, l'institution s'engouffre au contraire dans le tunnel des biotechnologies. Maquillées en issues de secours à la crise, ces dernières se voient octroyer dès 1982 le soutien du ministère de la Recherche et de l'Industrie à travers l'un de ses cinq programmes mobilisateurs ${ }^{17}$.

Quant au mouvement des boutiques de sciences, lancé en 1983 grâce à des financements ministériels permettant l'embauche de salariés, il tend à se déconnecter des mouvements sociaux, traitant principalement des demandes individuelles et n'influençant pas le cours des recherches. Répondant aux nouvelles missions des chercheurs d'œuvrer à la « diffusion de l'information scientifique et technique », il semble happé dans le grand tournant de la «

14 . S. Topçu, La France nucléaire. Gouverner une technologie contestée, La Découverte, Paris, 2013.

15. Cf., parmi d'autres, J.-P. Martin, Histoire de la nouvelle gauche paysanne. Des contestations des années 1960 à la Confédération paysanne, La Découverte, Paris, 2005.

${ }^{16}$. Collectif, Une agriculture du vivant. L'héritage de l'École de Beaujeu, Éditions du Fraysse/ CEREA, Monclar de Quercy, 2006.

17 . C. Bonneuil et F. Thomas, Gènes, profits, pouvoirs. Recherche publique et régimes de production des savoirs de Mendel aux OGM, Quæ, Versailles, 2009. 
médiation ». Parant à la connotation diffusionniste de la vulgarisation, la médiation fait office de fenêtre de captation institutionnelle des doutes et des critiques des scientifiques et de professionnalisation de certains acteurs. Le premier Centre de culture scientifique technique et industrielle (CCSTI) est créé à Grenoble en 1979, la Cité des sciences et de l'industrie voit le jour en 1986, date également de la création de l'association Les Petits Débrouillards. Les scientifiques accèdent à un nouveau rôle d'entrepreneurs culturels, dont la première place revient aux biologistes. Face au discrédit de la physique, ces derniers achèvent de conquérir l'espace médiatique et les nouveaux rayonnages des collections scientifiques grand public tandis que la biologisation du social accompagne le déclin du marxisme ${ }^{18}$.

Nouvel objet de consommation culturelle, les technosciences se présentent aussi comme un instrument privilégié de relance économique. Face aux limites de l'énergie fossile, la bioéconomie déploie ses promesses de renouveau par l'investissement dans l'information, la complexité et les biotechnologies. Amorcé sous la présidence de Valéry Giscard d'Estaing, un pilotage plus utilitariste de la recherche se met en place. Les lois d'orientation et de programmation de la recherche de 1982 et 1983 institutionnalisent les liaisons entre l'industrie et la recherche. Afin d'accroître les transferts vers les entreprises, des dispositifs spécifiques sont créés pour inciter les chercheurs à collaborer avec les industriels et favoriser la mobilité entre public et privé. L'innovation devient le maître mot des politiques de recherche, et les industriels sont associés à leur élaboration, grâce à la création des établissements publics à caractère scientifique et technologique (EPST) ${ }^{19}$. Obtenues en échange de la sécurisation des carrières (création d'un statut de chercheur), ces réformes parviennent à rallier la communauté scientifique à une vision " industrialisante » et militariste du progrès social. En créant de nouveaux intérêts corporatistes, la fonctionnarisation achète ainsi le calme social.

Cette reprise en main étatico-industrielle s'illustre dans le blocage de domaines de recherche à peine éclos (sur le solaire et les dangers des pesticides en particulier ${ }^{20}$ ) et dans la percée d'une stratégie du doute, au sein par exemple du Comité permanent pour l'amiante qui, dominé par les industriels, parvient à maintenir ce toxique dans la légalité.

Une dernière lecture de cet enterrement de la critique radicale des sciences fait valoir son intégration par le technocapitalisme converti au «nouvel esprit de la complexité et du réseau ${ }^{21} \gg$. Semblant marquer le dépassement de la big science fordiste discréditée, une large gamme de savoirs rompent en effet avec une conception fixiste et structuraliste de l'équilibre et de l'ordre pour embrasser un modèle plus fluide et adaptatif, attentif aux discontinuités, incertitudes, chaos et feedbacks. La modélisation et les simulations informatiques qui y contribuent rencontrent alors les aspirations à une science moins dogmatique et réductionniste, plus pragmatique et systémique. Pour

\footnotetext{
18 . S. Lemerle, Le singe, le gène et le neurone. Du retour du biologisme en France, PUF, Paris, 2014.

19 . J.-F. Théry et R. Barré, La loi sur la recherche de 1982. Origines, bilan et perspectives du " modèle français », INRA, Paris, 2001.

${ }^{20}$. F. Nicolino et F. Veillerette, Pesticides. Révélations sur un scandale français, Fayard/Pluriel,
} 
certains anciens critiques, le pari est réussi ; et il les engage dans un tournant libéral.

Emblématique de ces mutations, l'essor de la microélectronique parvient à dégager l'informatique de ses atours de guerre froide (origine militaire, outil de contrôle) et de productivité (appauvrissement du travail, chômage), et de l'hostilité qu'elle suscitait jusqu'à la fin des années 1970. Amorçant cette nouvelle phase d'industrialisation, le plan télématique de 1978 entreprend la colonisation des foyers, sous le regard sceptique de Terminal, dernière des revues de critique des technosciences. De l'« informatisation de la société » aux «nouvelles technologies », l'informatique joue ainsi « un rôle décisif dans l'écrasement des mouvements contestataires » et le « rétablissement d'un consensus autour du modèle économique dominant ${ }^{22} »$.

Alors que la " technoparade " de la nouvelle classe politique vise explicitement à enterrer le mouvement de critique radicale des sciences de la décennie précédente, la nouvelle génération de scientifiques critiques, confrontée à ce tournant industrialiste de la recherche, renoue avec une défense de la recherche fondamentale. La critique radicale de la décennie précédente devient inaudible. Parallèlement à ce repli, l'engagement au service du peuple se reconvertit en médiation auprès du grand public, selon un schéma strictement diffusionniste ou sous une forme plus « culturaliste » ${ }^{23}$. On comprend ainsi qu'à la fin de la décennie, Alexandre Grothendieck, qui s'est fait humble enseignant à l'université de Montpellier, entame une période de solitude et se retire alors à la campagne.

Paris, 2011 [2007].

21. C. Bonneuil, « Scientifiques, vie intellectuelle et espace public en France, 1962-2012 », dans

L. Jeanpierre et C. Charle, La vie intellectuelle en France, $x i x^{e}-x x i^{e}$ siècle, Seuil, à paraître.

22. C. Izoard, «L'informatique entre mise à feu et résignation », dans Les luddites en France.

Résistances à l'industrialisme et à l'informatisation, L'Échappée, Montreuil, 2010, p. 283 et 284.

23. J.-M. Levy-Leblond, Mettre la science en culture, Anais, Nice, 1986. 


\section{Des conflits des années 1990 au nouvel arsenal de la « démocratie technique »}

Les années 1990 sont le théâtre, à l'échelle européenne, d'un ensemble de conflits cristallisés autour de technologies, de scandales scientifiques et sanitaires, ou de grandes installations techniques : énergie nucléaire, semences et organismes génétiquement modifiés, sang contaminé, crise de la vache folle, nanotechnologies, etc. Contrairement aux années 1970, ces luttes sont souvent portées par des acteurs associatifs extérieurs aux milieux de la recherche : associations de malades, de défense de l'environnement, groupes antinucléaires, altermondialistes, etc. Ils sont " dotés d'une expertise scientifique et technique croissante ${ }^{21}$ ", et utilisent souvent les arènes judiciaires et médiatiques. Les scientifiques sont alors peu représentés dans ces luttes, si ce n'est sous la figure, souvent individuelle, du lanceur d'alerte, " plus focalisée sur des risques que sur des causes émancipatrices, moins encline à porter une critique sociale des institutions scientifiques ». Si les risques dominent les formes de critiques, ils forgent aussi les politiques publiques : les agences sanitaires se multiplient et l'expertise acquiert un rôle politique qu'elle ne quittera plus. Par ailleurs, les années 1990 sont aussi le produit des réformes des années 1980, dont l'esprit néolibéral se perpétue : la loi Allègre de 1999 encourage la création de start-up par les chercheurs, les projets de recherche obtiennent des financements s'ils sont porteurs de retombées industrielles, et le profil du chercheur technophile et « mercenaire de l'innovation » devient une figure médiatique normalisée ${ }^{22}$.

Dans ce contexte en tension, entre crise, contestation et innovation décomplexée, le tournant des années 2000 est porteur de redistributions des positions critiques à propos de la "démocratisation » des choix scientifiques et techniques. Ces redistributions (et les conflits qu'elles entraînent) s'organisent autour d'une nouvelle façon de nommer et de penser la critique des sciences : les relations « sciences-société ». L'Union européenne étant devenue grande mécène des recherches et ordonnatrice des politiques scientifiques, ces transformations ne se mènent dès lors plus seulement à l'échelle hexagonale.

Les conflits environnementaux et les nouvelles mobilisations ont grandement préoccupé les institutions européennes et nationales, qui raffinent leurs techniques de gouvernement pour les gérer. À l'échelle européenne, un arsenal d'instruments est alors développé afin de mesurer l'opinion publique concernant ces technologies et d'organiser le "public understanding of science " par des campagnes de communication et de médiation scientifique qui visent à redorer l'image des technosciences. En France, les CCSTI, les musées des sciences et de l'industrie ainsi que les initiatives de médiations scientifiques deviennent les bras armés de ces politiques. Ce mouvement

\footnotetext{
${ }^{21}$. Cette citation et la suivante, C. Bonneuil, « Scientifiques, vie intellectuelle et espace public en France », art. cité.

22. Ibid.
} 
politique, enclenché à la fin des années 1990, est très descendant et repose sur l'idée que les mobilisations sociales remettant en cause le dogme du progrès seraient le produit d'un manque de confiance lié à un manque de connaissance scientifique (conception que l'on nomme « modèle du déficit »).

Ces préoccupations institutionnelles ont été saisies par des chercheurs en sciences sociales aux ambitions réformatrices. Ce sont les nouveaux scientifiques « engagés », qui visent moins les dénonciations et les conflits que le consensus sciences-société. Ces chercheurs sont réunis autour du projet de « rapprocher la société civile de la programmation scientifique » et jouent un rôle de plus en plus important au sein des institutions. Ils rejettent le modèle du déficit et du public understanding of science, et contribuent à l'initiation d'un second mouvement politique au début des années 2000 centré sur les « relations sciences-société ». Ce modèle suggère que les conflits sociaux qui touchent aux sciences et aux technologies peuvent être résolus par la démocratisation des choix scientifiques et techniques et par des dispositifs dialogiques et participatifs. Le champ des science and technology studies (STS), à l'origine de ce diagnostic, a produit un vaste ensemble de théories normatives (démocratie technique, mode 2$)^{23}$ et de dispositifs participatifs destinés à porter ce programme (conférence de citoyens, constructive technology assessment) ${ }^{24}$. Les sociologues Charles Thorpe et Jane Gregory ont pointé les connivences de ce tournant participatif avec les politiques postfordistes alors en plein développement ${ }^{25}$. Au Royaume-Uni, les approches sciences-société sont ainsi façonnées dans des think tanks proches du Labour britannique à l'époque blairiste, et construites en articulation avec les modèles d' « économie de la connaissance » consuméristes et néolibérales.

Les théories sociales qui en émergent ont, par la suite, rapidement circulé dans des univers associatifs qui y ont adossé leur revendication, souvent par l'intermédiaire de chercheurs militants qui opèrent le rapprochement entre univers technocratique, académique et militant. À l'échelle française, on peut offrir une brève typologie des " militants sciences-société » qui illustre les variations politiques autour du thème de la démocratie technique :

- La critique antilibérale des technosciences : représentée par la Fondation Sciences Citoyennes qui défend une forme (radicale) de démocratie technique comme option de sortie des politiques technoscientifiques libérales. La Fondation revendique ainsi « l'accroissement des capacités de recherche et d'expertise de la société civile, des forces associatives, consuméristes, syndicales et citoyennes », «l'appui aux lanceurs d'alerte et le développement de controverses publiques et de "forums hybrides" sur les enjeux à forte technicité scientifique » et la promotion de "l'élaboration démocratique des choix scientifiques et techniques $»$;

\footnotetext{
23. M. Callon, P. Lascoumes et Y. Barthe, Agir dans un monde incertain, Essai sur la démocratie technique, Seuil, Paris, 2001 ; M. Gibbons, C. Limoges, H. Nowotny, S. Schwartzman, P. Scott et M. Troy, The New Production of Knowledge. The Dynamics of Science and Research in Contemporary Societies, Sage, Londres, 1994.

24 . Pour une vue d'ensemble, $c f$. D. Pestre, «Des sciences, des techniques et de l'ordre démocratique et participatif », Participations, vol. 1, n 1, 2011, p. 210-238, et C. Bonneuil et P.-B. Joly, Sciences, techniques et société, La Découverte, Paris, 2013.

25 . C. Thorpe et J. Gregory, « Producing the Post-Fordist Public. The Political Economy of Public Engagement with Science », Science as Culture, 2010, vol. 19, n³ 3, p. 273-301.
} 
- La réforme institutionnelle : représentée par l'Alliance Sciences Société, qui réunit des institutions scientifiques, des CCSTI et des associations. Toutes ces instances ne partageant pas la même position politique, l'Alliance vise à être un réseau très large de pression sciencessociété. En cherchant à se faire entendre « au plus haut niveau réglementaire et administratif par l'État ${ }^{26}$ ", l'Alliance s'approche peut-être le plus explicitement d'une stratégie réformatrice, cherchant à institutionnaliser encore plus fortement ses revendications ;

- Le dialogique professionnalisé : représenté par l'association VivAgora qui s'est professionnalisée dans l'organisation de dispositifs participatifs en partenariat avec des institutions publiques et privées. VivAgora oscille entre un positionnement en tant qu'organisation de la société civile aux affinités écologique et « citoyenniste», et un statut proche de celui d'une entreprise de conseil en débats publics ;

La technocratie « responsable 》 : représentée par l'Observatoire pour l'innovation responsable (OIR). Accueillie par l'École nationale supérieure des mines de Paris, l'OIR revendique « accompagner le mouvement vers une culture de responsabilité dans l'innovation, en se concentrant sur des secteurs spécifiques dans lesquels les défis de l'innovation responsable sont grands ${ }^{27}$ ", tels que la finance et les technologies de l'information et de la communication. L'OIR organise en effet des débats bien différents de ceux de VivAgora, principalement destinés à la rencontre de chercheurs, de membres d'administrations et d'institutions publiques, ainsi que d'entreprises. La position de l'Observatoire est donc celle d'un accompagnement de l'innovation : les logiques économiques et politiques qui la gouvernent actuellement sont maintenues mais elles sont responsabilisées, afin d'atteindre le « compromis positif» entre le progrès et ses impacts.

Les institutions ont rapidement fait usage de ces nouveaux acteurs et de leurs dispositifs participatifs lorsqu'elles étaient face à des critiques qu'elles ne parvenaient pas à gérer : l'INRA lors de la controverse des OGM, la communauté d'agglomération de Grenoble ou la région Île-de-France au moment des conflits sur les nanotechnologies, l'Agence nationale pour la gestion des déchets radioactifs (ANDRA) lors des conflits autour du centre de stockage Cigéo à Bure, etc. Après avoir été porteuses d'une critique à l'encontre des institutions au début des années 2000, les associations sciencessociété deviennent des ressources essentielles à de nombreux dispositifs institutionnels au tournant des années 2008-2009. Ce tournant est clairement celui des nanotechnologies, autour desquelles la machine participative fut peut-être la plus spectaculaire ${ }^{28}$.

Si les dispositifs de démocratie technique sont employés par les institutions pour réinsuffler une légitimité à leurs politiques technoscientifiques, cette institutionnalisation transforme l'arène académico-associative sciencessociété en retour : elle a pour effet de spécialiser, voire de professionnaliser, les

\footnotetext{
${ }^{26}$. Charte de l'Alliance Sciences Société, <alliance-sciences-societe.fr/charte>.

27 . Site Internet de l'Observatoire de l'innovation responsable, <www.debatinginnovation.org $>$.

${ }^{28}$. Simples Citoyens, « Nanotechnologies / Maxiservitude. Des contributions grenobloises à l'automatisation du cheptel humain », 2003, <www.piecesetmaindoeuvre.com> ; B. Laurent, Democracies on Trial. Assembling Nanotechnologies and its Problems, thèse de doctorat en socioéconomie de
} 
acteurs académiques et ceux de la société civile, autant que de standardiser les dispositifs et revendications. Le régime sciences-société s'est ainsi trouvé stabilisé entre les années 2000 et 2010 par la standardisation d'une certaine conception des conflits portant sur les technologies, ainsi que par la légitimation de l'expertise de certains chercheurs en sciences sociales reconnus comme spécialistes pour organiser ces dialogues. La multiplication des dispositifs de démocratie technique rend ainsi compte des formes de cooptation des associations et des nouvelles stratégies institutionnelles de gestion des conflits.

La démocratie technique est toutefois contestée par différents groupes, tels que Pièces et Main d'Euvre (PMO) ou, de l'intérieur du monde académique, le Groupe Oblomoff. Ces derniers considèrent qu'il n'est pas possible de démocratiser les technosciences, et que la démocratie technique est un artifice servant à rendre acceptables les choix technologiques pris par les pouvoirs. Le militantisme sciences-société revendique l'inclusion des citoyens et le désenclavement des choix scientifiques et techniques, alors que les critiques radicales des technologies (et de leur démocratisation) refusent la logique de l'expertise (et donc de la « contre-expertise » ou de «l'expertise profane ») et son extension à de nombreuses formes de la vie politique et sociale ${ }^{32}$. L'arène sciences-société et la critique radicale des technosciences sont séparées par une ligne de conflit construite autour de la démocratie technique. La première y voit un espace d'émancipation quand la seconde la considère comme un ersatz de démocratie et un nouvel instrument d'aliénation. À partir du milieu des années 2000, différentes actions militantes (perturbation de débats, pamphlets) sont menées contre les dispositifs sciences-société et ses acteurs. PMO rebaptise les associations dialogiques professionnalisées (comme VivAgora) d' " entreprises de communication événementielle », celles de la critique antilibérale de « tentacule vert» (Fondation Sciences Citoyennes), et les sociologues de " mercenaires " et d'" acceptologues ". L'institutionnalisation des militants et des chercheurs est donc critiquée par des groupes plus radicaux, qui rappellent bien plus les registres contestataires de la critique des sciences des années 1970 qu'ils ne s'apparentent au militantisme sciences-société.

l'innovation, École nationale supérieure des mines de Paris, 2011.

32. Groupe Oblomoff, Un futur sans avenir. Pourquoi il ne faut pas sauver la recherche scientifique, L'Échappée, Montreuil, 2009 ; Pièces et Main d'Euvre, « Participer c'est accepter », septembre 2009, $<$ www.nanomonde.org/Participer-c-est-accepter $>$. 


\section{Conclusion}

La remise en cause du consensus national autour des « retombées positives » de la recherche scientifique durant les années 1970 n'aura été que de courte durée. Ce moment fut pourtant fondateur, tant pour l'engagement d'Alexandre Grothendieck que pour l'analyse du rôle des technosciences dans l'essor et le maintien d'une société de production-consommation de masse, qui marqua la naissance de l'écologie.

$\mathrm{Au}$ fil des réformes, des années 1980 aux années 2000, le milieu scientifique semble avoir profondément intériorisé les injonctions industrialoétatiques à la croissance nationale et à la compétitivité internationale, comme en témoignent les formes dominantes d'engagement actuel des chercheurs, du mouvement Sauvons la recherche à Sciences en marche. Malgré la réflexion en cours dans certaines branches syndicales minoritaires, l'autocritique de la science, menée de l'intérieur des institutions, reste aujourd'hui extrêmement délicate et marginale. Les rares scientifiques critiques investissent à leur tour un militantisme "sciences-société ", lequel leur offre un espace parallèle d'engagement. Pourtant, face au durcissement des rapports de forces qui laisse présager la fin des débats publics et révèle la fragilité des dispositifs de démocratie technique, la contestation de cette dernière semble gagner en audience, en particulier dans les milieux décroissants ou d'écologie radicale.

Aussi ce tournant semble-t-il propice à une réappropriation de l'héritage critique de Grothendieck, laquelle a déjà été engagée par les mouvements actuels de critique radicale des technosciences ${ }^{29}$. Une telle relecture militante du mouvement de critique radicale des sciences des années 1970, nourrie du récit donné par les acteurs ${ }^{30}$ et de quelques travaux universitaires sur le sujet ${ }^{31}$, viendrait alors sûrement enrichir les formes actuelles de la contestation.

Céline Pessis mène des recherches sur l'histoire des mouvements écologistes. Elle a coordonné Survivre et Vivre. Critique de la science, naissance de l'écologie (L'Échappée, 2014) et, avec Sezin Topçu et Christophe Bonneuil, Une autre histoire des "Trente Glorieuses ». Modernisation, contestations, pollutions (La Découverte, 2013).

Sara angeli aguiton est sociologue. Elle a consacré sa thèse à l'étude de l'émergence de la biologie synthétique en France et aux États-Unis, suivant les politiques de développement et de régulation qui illustrent les formes actuelles du «progrès » technique. Elle travaille aujourd'hui sur l'économie politique du changement climatique, saisie par les assurances des catastrophes naturelles.

\footnotetext{
${ }^{29}$. Cf., par exemple, B. Louart, « Quand les scientifiques critiquaient la science... », Offensive, ${ }^{\circ}$ 11, mai 2006.

30. Ce premier moment d'écriture de l'histoire fut favorisé par le renouveau contestataire des années 1990. Cf. P. Petitjean, « La critique des sciences en France », art. cité ; J. Peiffer, « Les débuts de la critique féministe des sciences en France (1978-1988)», dans D. Gardey et I. Löwy (dir.), L'invention du naturel. Les sciences et la fabrication du féminin et du masculin, Éditions des archives contemporaines, Paris, 2000, p. 75-86.

${ }^{31}$. Ce second moment historiographique accompagna l'essor du militantisme « science-société » dans les années 2000. $C f$. <www.science-société.fr>.
} 